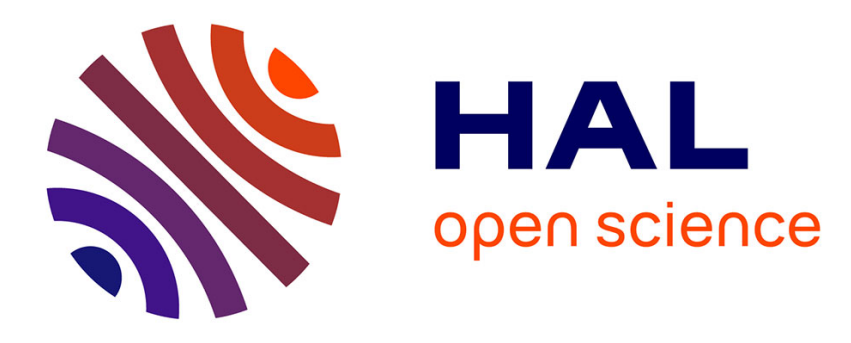

\title{
Spectral Photon-Counting Computed Tomography for Coronary Stent Imaging
}

Grischa Bratke, Tilman Hickethier, Daniel Bar-Ness, Alexander Christian

Bunck, David Maintz, Gregor Pahn, Philippe Coulon, Salim Si-Mohamed, Philippe Douek, Monica Sigovan

\section{To cite this version:}

Grischa Bratke, Tilman Hickethier, Daniel Bar-Ness, Alexander Christian Bunck, David Maintz, et al.. Spectral Photon-Counting Computed Tomography for Coronary Stent Imaging. Investigative Radiology, 2020, 55 (2), pp.61-67. 10.1097/RLI.0000000000000610 . hal-03097180

\section{HAL Id: hal-03097180 https://hal.science/hal-03097180}

Submitted on 5 Jan 2021

HAL is a multi-disciplinary open access archive for the deposit and dissemination of scientific research documents, whether they are published or not. The documents may come from teaching and research institutions in France or abroad, or from public or private research centers.
L'archive ouverte pluridisciplinaire HAL, est destinée au dépôt et à la diffusion de documents scientifiques de niveau recherche, publiés ou non, émanant des établissements d'enseignement et de recherche français ou étrangers, des laboratoires publics ou privés. 


\section{Spectral photon counting CT for coronary stent imaging: Evaluation}

\section{of the potential clinical impact for the delineation of in-stent}

\section{restenosis}

Grischa Bratke, MD, Tilman Hickethier, MD, Daniel Bar-Ness, PhD, Alexander Christian Bunck, MD, David Maintz, MD, Gregor Pahn, Philippe Coulon, PhD, Salim Si-Mohamed, MD, Philippe Douek, MD, PhD, and Monica Sigovan, PhD

\section{Abstract}

Objectives:

In-stent restenosis (ISR) is one of the main long-term complications following coronary stent placement and the ability to evaluate ISR non-invasively using coronary computed tomography (CT) angiography remains challenging. For this application, spectral photon counting CT (SPCCT) has the potential to increase image quality and reduce artifacts due to its advanced detector technology.

Our study aimed to verify the technical and clinical potential of a novel SPCCT prototype using an ISR phantom setup.

Materials and Methods:

Soft plaque-like restenosis (45 HU; approx. $50 \%$ of the stent lumen) were inserted into 10 different coronary stents ( $3 \mathrm{~mm}$ diameter), which were placed in a vessel phantom and filled with a contrast agent $(400 \mathrm{HU})$. A research prototype SPCCT and a clinical dual-layer CT (DLCT) (IQon; Philips) with comparable acquisition and reconstruction parameters were used to scan the phantoms. Conventional polyenergetic (PolyE) and monoenergetic (MonoE) images with 4 different energy 
levels $(40,60,90,120 \mathrm{keV})$ were reconstructed. Qualitative (delineation of the stenosis and adjacent residual lumen using a 5-point Likert scale) and quantitative (image noise, visible lumen diameter, lumen diameter adjacent to the stenosis, contrast-to-noise ratio of the restenosis) parameters were evaluated for both systems.

Results:

The qualitative results averaged over all reconstructions were significantly superior for SPCCT compared to DLCT (e.g. subjective rating of the best reconstruction of each scanner: DLCT PolyE: $2.80 \pm 0.42$ versus SPCCT MonoE $40 \mathrm{keV}$ : $4.25 \pm 1.03$ ). Stenosis could be clearly detected in 9 and suspected in 10 of the 10 stents with both SPCCT and DLCT. The residual lumen next to the stenosis was clearly delineable in 7 out of 10 stents $(0.64 \pm 0.11 \mathrm{~mm}$ or $34.97 \%$ of the measured stent lumen) with SPCCT while it was not possible to delineate the residual lumen for all stents using DLCT. The measured diameter of the lumen within the stent was significantly higher for SPCCT compared to DLCT in all reconstructions with the best results for the MonoE $40 \mathrm{keV}$ images (SPCCT: $1.80 \pm 0.17 \mathrm{~mm}$; DLCT: $1.50 \pm 0.31 \mathrm{~mm}$ ). The image noise as well as the contrast-to-noise ratio were better for DLCT than for SPCCT (Contrast-to-noise ratio: DLCT MonoE 40: $31.58 \pm 12.54$; SPCCT MonoE 40: $4.64 \pm 1.30)$.

Conclusion:

SPCCT allowed for the non-invasive evaluation of ISR with reliable results regarding the residual lumen for most tested stents and the clear identification or suspicion of 
stenosis for all stents. In contrast, the residual lumen could not be detected for a single stent using DLCT.

\section{Key words:}

Photon-counting, Dual Energy, Computed Tomography, CT, Stent, Cardiac, Dual Layer, Coronary, Restenosis, Artery 


\section{Introduction}

Replacing invasive cardiac catheter examinations by non-invasive coronary computed tomography angiography (CCTA) scans is very desirable due to the risk for potential adverse events of cardiac catheterization (1.7\% for a severe complication and $0.1 \%$ for mortality). ${ }^{1}$ However, the application of CCTA is partially limited due to technical reasons, especially beam hardening and blooming. ${ }^{2}$ Several recent technical improvements, including different reconstruction algorithms $s^{3}$ and spectral imaging, ${ }^{4}$ have led to increased accuracy of stenosis quantification mainly by artifact reduction. However, the exact evaluation of stent lumen and the delineation of possible in-stent restenosis (ISR) remains challenging with a significant number of unsatisfying ISR evaluation results. Eckert et al. found $25 \%$ false positive or inconclusive findings ${ }^{5}$ and $\mathrm{Li}$ et al. a positive predictive value of $82.2 \%$ for all stents and only $68.2 \%$ for small caliber stents ${ }^{6}$.

Novel spectral photon counting CT (SPCCT) systems equipped with photon counting detectors (PCD) can overcome some of the technical shortcomings associated with CCTA due to two inherently different technological aspects. PCDs have a continuous sensor layer which converts the incoming $\mathrm{x}$-ray photon directly into electrical charges. ${ }^{7}$ The direct conversion of the photons makes the use of optical nontransparent separators unnecessary and results in an increased dose-efficiency and smaller pixel size of the detector. The incoming photons are also spectrally discriminated with pulse height analysis in multiple energy windows and therefore offer high quality spectral capabilities. ${ }^{8}$ Recent studies have already shown improvements in image quality of in-vitro stent imaging for PCD scanners with better in-stent lumen delineation and reduced blooming artifacts. ${ }^{9-11}$ However, from a clinical 
perspective the question remains, whether these incremental advancements result in a real change of diagnostic capabilities, namely the ability to delineate both, the stenosis itself and the adjacent residual lumen. If so, this option for a non-invasive exact quantification or exclusion of in-stent stenosis could truly reduce the number of diagnostic catheterizations and therefore decrease unnecessary adverse events.

This in-vitro study compared the visibility of artificial in-stent stenosis in different coronary stents using spectral dual-layer CT (DLCT) and SPCCT.

\section{Materials and Methods:}

Phantom

A straight plastic tube with a $3 \mathrm{~mm}$ diameter was used as a coronary artery phantom. The material had a wall thickness of $0.3 \mathrm{~mm}$ with an attenuation comparable to a vessel wall (35 Hounsfield Units $(\mathrm{HU})$ ). Ten different stents (Table 1) made of five different materials were used to account for the broad variety of commercially available stents and to verify the clinical reliability. The stents were placed in the middle of the plastic tube with an average length of $20.3 \mathrm{~mm}( \pm 5.18 \mathrm{~mm})$ and a strut thickness of $0.13 \mathrm{~mm}( \pm 0.15 \mathrm{~mm})$. The artificial hypodense stenoses were made of a wax-based material mixed with ethiodized oil (Lipiodol Ultra-Fluid; Guerbet $\mathrm{GmbH}$, Sulzbach, Germany) titrated to measure $45 \mathrm{HU}$ at $120 \mathrm{kVp}$. This was chosen because in stented lesions the late lumen loss and restenosis are mainly the result of neointimal tissue proliferation with similar densities ${ }^{12}$. The grain-shaped restenoses were positioned angiographically-guided inside the stented tube lumen. To calibrate each stenosis to $50 \%$ of the total diameter, the stenosis was passed with a $1.5 \mathrm{~mm}$ balloon-catheter (Armada 14; Abbott $\mathrm{GmbH}$, Wiesbaden, Germany) using a 
microwire (V-14 Control Wire; Boston Scientific GmbH, Ratingen, Germany). After verification of the correct position, the balloon was inflated to its nominal pressure. The tube was filled with the iodine-based contrast agent lohexal $(300 \mathrm{mg}$ lohexol/mL; Accupaque 300; GE Healthcare GmbH, Solingen, Germany) which was diluted with saline solution to a density of $400 \mathrm{HU}$ at $120 \mathrm{kV} / 100 \mathrm{mAs}$ using DLCT. The tube was placed in a plastic container $(36 \times 24 \mathrm{~cm})$ which was fluid-filled (Sodium chloride). The phantoms were placed in the isocenter of each scanner parallel to the z-axis.

\section{CT acquisition and reconstruction parameters}

The acquisition parameters for both CT scanners were chosen to keep the differences between the systems as minimal as possible (Table 2).

The SPCCT prototype used a cadmium zinc telluride-based PCD. The highest possible resolution was chosen with a collimation of $9 \times 0.25 \mathrm{~mm}$ and a slice thickness of $0.25 \mathrm{~mm}$. Tube current was set to $100 \mathrm{mAs}$ with $120 \mathrm{kVp}$ and a rotation time of $1 \mathrm{~s}$. The images were reconstructed with a sharp filter, an image matrix of 512 x 512 pixels and a field of view (FOV) of $102 \mathrm{~mm}$. Conventional polyenergetic (PolyE) and four different monoenergetic (MonoE) images (40, 60, 90, $120 \mathrm{keV}$ ) were reconstructed. For the used SPCCT prototype only filtered back projection was available as reconstruction method without any iterative reconstruction algorithms. Further technical details concerning this prototype system are provided in previous publications. ${ }^{13,14}$

For comparison, dual energy CT data was acquired on a 128-row DLCT scanner (IQon, Philips Healthcare, Best, The Netherlands). Acquisition parameters for the DLCT were a collimation of $64 \times 0.625 \mathrm{~mm}$ with the thinnest possible slice thickness 
of $0.67 \mathrm{~mm}$ and a rotation time of $0.27 \mathrm{~s}$. This also represents our current clinical standard for CCTA. In line with SPCCT parameters, the tube current was set to 100 mAs with $120 \mathrm{kV}$ without automatic dose modulation. The images were calculated with a dedicated cardiac stent reconstructions filter and the iterative reconstruction components set to level 0 . This was the most comparable setting that could be achieved for DLCT as plain filtered back projection without any iterative component was not available on this commercial system.

\section{Data analysis}

Multiplanar reformats (MPR) in coronary, axial and sagittal orientation were reconstructed on the same offline workstation for all images (IntelliSpace Portal; Philips Healthcare, Best, The Netherlands) with a thickness equal to that of the underlying slice stack. Two raters (R1 and R2, with 6 and 5 years of experience in cardiovascular imaging, respectively) performed an independent quantitative and qualitative analysis of all available images.

\section{Quantitative analysis}

In-stent diameter and visible residual lumen next to the stenosis were measured by both raters with an electronic caliper tool (Figure 1). Reader 2 additionally measured the attenuation in Hounsfield Units (HU) with standardized regions of interest (ROIs) in the tube lumen outside the stent ( $\mathrm{ROI} 1)$, in the stent ( $\mathrm{ROI} 2)$, and in the stenosis (ROI 3) as well as the standard deviation of the attenuation outside the tube in the surrounding container (ROI 4) (Figure 2). The difference in the stenosis density was calculated as that between the density of the stenosis $(\mathrm{ROI} 3)$ and the density inside the stent beside the stenosis ( $\mathrm{ROI} 2)$. The in-stent attenuation difference between the lumen outside ( $R O \mathrm{I}_{1}$ ) and within the stent ( $\mathrm{ROI} 2$ ) was calculated by subtracting the corresponding ROls accordingly. To avoid potential inter-reader differences due to 
different window settings, predefined parameters for the window center and width for each reconstruction were used. The window level was equal to the density of the ROI measurement in the tube volume outside the stent (ROI 1) for each reconstruction. According to previous findings, ${ }^{15}$ the window width was then set to a multiplication of the level value, which was set to a factor of 2.5 based on a consensus reading. The diameter was determined next to the end of the stent in the coronal and sagittal reformation (average of both measurements). At the middle of the stenosis, the size of the residual lumen was measured on the coronal, sagittal, and axial reformations. Due to the eccentric shape and position of the artificial stenosis the average of the two largest diameters was used. The standard deviation of the density in the surrounding liquid (ROI 4) served as a parameter for the image noise. The contrastto-noise ratio $(\mathrm{CNR})$ of the stenosis was calculated as the difference in the stenosis density divided by the image noise.

\section{Qualitative analysis}

Both readers evaluated the subjective visibility of the stenosis and the remaining lumen on the coronal, axial and longitudinal MPR images using a 5-point Likert scale (1: image quality impedes lumen assessment and stenosis not visible; 2: lumen appears stenosed with unclear extent; 3: stenosis and extent clear, but residual lumen undistinguishable; 4: stenosis clear and residual lumen distinguishable; 5: stenosis and residual lumen clear).

\section{Statistical analysis}

Statistical analyses were performed using GraphPad Prism Version 8.0.2 (GraphPad Software Inc., San Diego, California, USA). All data are reported as the mean \pm standard deviation (SD). For the ordinal data of the subjective reading, the Friedman 
test was used, followed by Dunn's multiple comparisons post hoc test. The different quantitative parameters with continuous data were tested for significance with a oneway analysis of variance (ANOVA), Tukey test for post hoc analysis for the different reconstructions for a single scanner and the Bonferroni test for the comparison of the same reconstruction between different scanners. A p-value of $<0.05$ was considered statistically significant. The interrater agreement for the in-stent and residual lumen measurements was tested with the Pearson correlation coefficient and the subjective scoring with Cohen's kappa. According to Landis and Koch, ${ }^{16}$ values of 0.61 to 0.80 were interpreted as substantial, and 0.81 to 1.00 , as almost perfect agreement.

\section{Results}

\section{Lumen assessment}

The interrater agreement between both the raters was almost perfect for measurements of the in-stent lumen $(r=0.97)$ and residual lumen next to the stenosis $(r=0.99)$. The average measured lumen of the stents for all reconstructions was significantly higher for SPCCT than for DLCT $(p=0.001)$. The highest measured lumen for SPCCT was $1.83 \pm 0.17 \mathrm{~mm}$ in MonoE $40 \mathrm{keV}$ images and $1.50 \pm 0.31 \mathrm{~mm}$ for the DLCT with MonoE $40 \mathrm{keV}$ (Figure 3, Table 3). Despite the clear trend for a higher measured lumen in the low energetic MonoE images, there was no statistically significant difference between the different reconstructions within each scanner.

The residual lumen was not visible in any reconstruction of DLCT images and therefore, the measured residual lumen was $0 \mathrm{~mm}$ in all DLCT reconstructions and stents. For SPCCT, the best delineation of the residual lumen was achieved with the PolyE reconstructions with an average measured diameter of $0.45 \pm 0.32 \mathrm{~mm}$. 
Among different SPCCT reconstructions, the results were significantly better for the PolyE $(p=0.011)$, MonoE $40 \mathrm{keV}(p=0.015)$ and MonoE 60 images $(p=0.018)$ than for the high energetic MonoE $120 \mathrm{keV}$ images (Figure 4, Table 3). In addition, the residual lumen was rated as distinguishable in at least one reconstruction in seven out of the ten stents. The average residual lumen of these seven stents was $0.64 \pm$ $0.11 \mathrm{~mm}$ in the PolyE reconstruction, which is equal to $34.97 \%$ of the measured total lumen or $43 \%$ of the real residual lumen.

ROI-based density analysis

For the Wiktor stent the artifacts due to beam hardening affected the image quality so much that the exact extent of stenosis could not be detected on any reconstruction for both scanners, making a reliable measurement of the attenuation in the stent and stenosis impossible. To avoid the potential influence of a wrongly placed measurement, the stent was excluded from the following ROI-based analysis.

In line with previous dual energy studies, the attenuation within the tube lumen differed significantly depending on the chosen reconstruction with the highest attenuation for the low keV MonoE images and the lowest for the high keV MonoE images (Suppl. Table 1 for detailed results). The attenuation was higher within the stent ( $\mathrm{ROI} 2$ ) compared to the $\mathrm{ROI}$ outside the stent ( $\mathrm{ROI} 1)$ for both scanners due to beam hardening caused by the surrounding stent material. Averaged over all reconstructions the SPCCT showed a significant smaller in-stent attenuation difference $(p=0.025)$; however, none of the individual reconstructions were superior compared to other reconstructions of the same scanner or the same reconstruction on the other scanner. For SPCCT the MonoE $40 \mathrm{keV}(67.31 \pm 45.48 \mathrm{HU})$ showed the largest difference, with the smallest observed in the MonoE $120 \mathrm{keV}$ images (48.88 \pm 82.88 $\mathrm{HU}$ ). For DLCT however, the MonoE $40 \mathrm{keV}$ images had a smaller difference 
$(94.78 \pm 51.48)$ than the MonoE $120 \mathrm{keV}$ images $(178.49 \pm 306.27)$, and the PolyE images provided the lowest in-stent attenuation difference $(69.20 \pm 39.45 \mathrm{HU})$ (Suppl. Table 1). The high variability observed with DLCT was caused mainly by the Tantal stent and it's artifacts within the stent lumen (ROI 2).

Overall, stenosis density differences were significantly higher for DLCT than SPCCT $(p<0.001)$. When comparing the individual reconstructions of both systems, the MonoE $40 \mathrm{keV}(p<0.001)$ and MonoE $60 \mathrm{keV}$ images $(p=0.005)$ of the DLCT were superior to the corresponding SPCCT images. For both scanners the MonoE $40 \mathrm{keV}$ images were superior over all other reconstructions (all $p \leq 0.001)$ (Table 4).

The noise measured in the fluid next to the stents was significantly higher for SPCCT than DLCT (all p < 0.001; e.g. for MonoE $40 \mathrm{keV}$ : SPCCT158.47 $\pm 24.29 \mathrm{HU}$, DLCT $25.79 \pm 9.31 \mathrm{HU})($ Table 4). There were no significant differences in the image noise for the different DLCT reconstructions. For SPCCT however, image noise was significantly higher (all $p<0.05)$ in low (MonoE 40) and high (MonoE 90 and MonoE 120) energetic MonoE reconstructions compared to the PolyE and $60 \mathrm{keV}$ MonoE images (which had comparable noise levels).

Due to the higher stenosis density differences and lower image noise, CNR was significantly higher in DLCT images than in the corresponding SPCCT images $(p<$ 0.001) (Table 5). MonoE $40 \mathrm{keV}$ DLCT images showed the highest overall CNR values (31.18 \pm 13.57), which were significantly higher than in any other reconstruction (all $p<0.05$ ). For SPCCT, the PolyE images offered the best CNR with a mean value of $8.67 \pm 2.25$.

Qualitative analysis

The interrater agreement for the subjective scoring was almost perfect with a kappa of 0.84 . Throughout every reconstruction the qualitative analysis showed superior 
scores for SPCCT (Figure 5 and 6) with an overall significantly better subjective rating $(p<0.001)($ Table 5). There was a visible trend to higher scores for SPCCT images of the corresponding reconstructions of both scanners, which did not reach statistical significance, e.g. SPCCT MonoE $60 \mathrm{keV}$ with $3.95 \pm 1.46$ and DLCT MonoE $60 \mathrm{keV}$ with $2.5 \pm 0.71(p=0.60)$. MonoE $40 \mathrm{keV}$ resulted in the best rating for SPCCT with $4.25 \pm 1.03$ compared to PolyE as the best reconstruction for DLCT at $2.80 \pm 0.42(p=0.90)$. For both scanners, the stenosis was clearly detectable in 9 out of 10 stents (rating $\geq 3$ ) and suspected in all stents (rating $\geq 2$ ). 


\section{Discussion}

The use of SPCCT for stent imaging offered a superior subjective image quality which can result in completely new diagnostic capabilities and may potentially enable additional applications for CCTA. A clear evaluation of the in-stent stenosis and adjacent residual lumen was possible and allowed for highly reliable non-invasive assessments of the actual extent of stenosis in nearly all tested stents. The PolyE images showed a good overall delineation for the different materials, and the spectral MonoE images allowed for an additional reduction of stent-related artifacts and better visualization of the stent lumen. Potential stenoses could be detected for all the tested stents using the low-energetic MonoE reconstructions, and a clear visualization of ISR was possible in $90 \%$ of cases, whereas the PolyE images were occasionally impaired by artifacts of the stent material (especially by Tantalum), and stenosis was suspected in only $80 \%$ of cases.

The parameters for DLCT were selected to align as closely as possible to SPCCT. Therefore, further improvements in DLCT image quality by utilizing optimized acquisition and reconstruction parameters might be possible - however, the detector resolution would still be the limiting factor. Additionally, our results with a clear detection of the stenosis for $80 \%$ of the tested stents and a suspicion in $90 \%$ of the cases are in line with previous studies testing the diagnostic quality of different CT systems; ${ }^{5,6,17}$ thus, the influence of our used acquisition and reconstruction parameters on the overall results is likely limited. For both systems, the thinnest possible slice was chosen which resulted in the SPCCT thickness being less than half the thickness of DLCT. The thinner slice thickness caused increased noise for the SPCCT images. This is supported by the results from another in-vitro stent study by Almutairi et al., which found that image noise increased by a factor of 2.7 when 
the slice thickness was reduced from 1.5 to $0.67 \mathrm{~mm} .{ }^{18}$ On the other hand, the thinner slice thickness might assist with the discrimination of smaller structures and reduces potential partial volume effects that could occur in an in-vivo setting and could therefore increase the effective noise level. Specifically, an eccentric position of the stent with respect to the z-axis increases partial volume effects of the stent material within the lumen, which can only be reduced by a thinner slice thickness. The used prototype SPCCT is only capable of using filtered back projection. Further developments in SPCCT image processing are expected to reduce image noise for this system and would therefore potentially allow for the achievement of better CNR values than DLCT. Spiczak et al. have already shown that the application of an iterative reconstruction algorithm for a SPCCT prototype of a different vendor could decrease the image noise by 41 to $59 \% .{ }^{10} \mathrm{~A}$ comparable reduction would reduce the noise level of SPCCT even below DLCT values for some reconstructions. In line with previous results of Hickethier et al., ${ }^{19}$ we found a significant reduction of blooming artifacts with the application of higher energy levels. However, adjusting window settings to the individual attenuation of each reconstruction did not led to an increase in the overall measured lumen in our study; however, there was even a trend towards higher values for low keV images observed. For SPCCT, the measured lumen was significantly closer to the real stent diameter of $3 \mathrm{~mm}$ compared to DLCT. The identification of stenoses was enhanced by using lower energy levels due to the increased attenuation difference between the hypodense stenotic material and the contrast agent in the adjacent vessel lumen.

While drug-eluting stents significantly reduce the number of in-stent stenoses, ${ }^{20}$ these are still highly relevant complications ${ }^{21}$ which adversely affect clinical outcomes. ${ }^{22,23}$ Bossi et al. found the pattern of the in-stent stenosis as well as the 
time to in-stent restenosis $<90$ days to be independent predictors for subsequent revascularization. ${ }^{24}$ Therefore, the possibility for non-invasive assessment and exact measurement of the stenosis would be highly desirable, especially since ISR can be diagnosed without specific symptoms. ${ }^{16}$ Future screening with SPCCT might be an option to identify ISR and select patients in need of angioplasty. This is especially valuable since the application of non-invasive diagnostic tests not only reduces the amount of potential side effects, but also reduces the costs per patient. Min et al. found that the use of CCTA (assuming 90\% sensitivity and 96\% specificity) for patients with suspected in-stent restenosis can reduce the cost per stented patient to less than $30 \%$ of the invasive strategy with direct referral to invasive coronary angiography for all patients (\$490 US vs. $\$ 1656$ US) ${ }^{25}$

The main limitation of this study was the in-vitro design as a proof-of-concept. Possible motion artifacts in patients could result in inferior image quality. In addition, all stents had a diameter of $3 \mathrm{~mm}$ which was considered as the lower limit for large caliber stents by $\mathrm{Li}$ et al. with better accuracy. ${ }^{6}$ Additional studies need to test whether the lumen will be still visible for stents with smaller diameters. The grade of the stenosis was overrated on average with $65 \%$ instead of the true $50 \%$ for the stents with a subjective scoring of 4 or better. Calcification of the imitated vessel was completely absent which might influence the overall image quality or stenosis delineation; however, spectral reconstructions already showed to have the potential of reducing the related artifacts as well (similar to the stent artifacts). ${ }^{4}$ Additionally, in our study a clinically desirable concentration of the contrast agent within the vessel phantom was chosen to evaluate the performance potential. Results with suboptimal contrast conditions might be different and should be evaluated in further studies. 
For non-invasive detection of ISR, we were able to demonstrate for the first time that the technical improvements of SPCCT yields clinically relevant additional information. The combination of higher resolution and spectral information enabled the detection or suspicion of an ISR in all tested stents. Therefore, SPCCT systems have the potential to reduce the need for invasive coronary angiographies with primary diagnostic purposes. 


\section{References}

1. Noto TJ, Johnson LW, Krone R, et al. Cardiac catheterization 1990: a report of the registry of the society for cardiac angiography and interventions (SCA\&I). Cather Cardio Diag. 1991;24(2):75-83.

2. Maintz D, Juergens K-U, Wichter T, et al. Imaging of coronary artery stents using multislice computed tomography: in vitro evaluation. Eur Radiol. 2003;13(4):830-835.

3. Puchner SB, Ferencik M, Maehara A, et al. Iterative Image reconstruction improves the accuracy of automated plaque burden assessment in coronary CT angiography: a comparison with intravascular ultrasound. Am J Roentgeno. 2017;208(4):777-784.

4. Symons R, Choi Y, Cork TE, et al. Optimized energy of spectral coronary CT angiography for coronary plaque detection and quantification. J Cardiovas Comput. 2018;12(2):108-114.

5. Eckert J, Renczes-Janetzko P, Schmidt M, et al. Coronary CT angiography (CCTA) using third-generation dual-source CT for ruling out in-stent restenosis. Clin Res Cardiol. 2018. Available at: https://doi.org/10.1007/s00392-018-1369-1. Accessed January 27, 2019.

6. Li Y, Yu M, Li W, et al. Third generation dual-source CT enables accurate diagnosis of coronary restenosis in all size stents with low radiation dose and preserved image quality. Eur Radiol. 2018;28(6):2647-2654.

7. Taguchi K. Energy-sensitive photon counting detector-based X-ray computed tomography. Radiol Phys Technol. 2017;10(1):8-22.

8. Taguchi K, Iwanczyk JS. Vision 20/20: Single photon counting x-ray detectors in medical imaging. AIP Conf Proc. 2013;40(10):100901.

9. Mannil M, Hickethier T, von Spiczak J, et al. Photon-counting CT: high-resolution imaging of coronary stents. Invest Radiol. 2018;53(3):143-149.

10. von Spiczak J, Mannil M, Peters B, et al. Photon counting computed tomography with dedicated sharp convolution kernels: tapping the potential of a new technology for stent imaging. Invest Radiol. 2018;53(8):486-494.

11. Symons R, De Bruecker Y, Roosen J, et al. Quarter-millimeter spectral coronary stent imaging with photon-counting CT: Initial experience. J Cardiovasc Comput. 2018;12(6):509515.

12. Hoffmann Rainer, Mintz Gary S., Dussaillant Gaston R., et al. Patterns and mechanisms of in-stent restenosis. Circulation. 1996;94(6):1247-1254.

13. Kopp FK, Daerr H, Si-Mohamed S, et al. Evaluation of a preclinical photon-counting CT prototype for pulmonary imaging. Sci Rep. 2018;8. Available at: https://www.ncbi.nlm.nih.gov/pmc/articles/PMC6255779/. Accessed April 29, 2019.

14. Si-Mohamed S, Thivolet A, Bonnot P-E, et al. Improved peritoneal cavity and abdominal organ imaging using a biphasic contrast agent protocol and spectral photon counting computed tomography K-Edge Imaging. Invest Radiol. 2018;53(10):629-639. 
15. Doerner J, Luetkens JA, Iuga A-I, et al. Poly-energetic and virtual mono-energetic images from a novel dual-layer spectral detector CT: optimization of window settings is crucial to improve subjective image quality in abdominal CT angiographies. Abdom Radiol. 2018;43(3):742-750.

16. Landis JR, Koch GG. The measurement of observer agreement for categorical data. Biometrics. 1977;33(1):159-174.

17. Yang J, Yang X, De Cecco CN, et al. Iterative reconstruction improves detection of instent restenosis by high-pitch dual-source coronary CT angiography. Sci Rep. 2017;7(1):6956.

18. Almutairi AM, Sun $\mathrm{Z}, \mathrm{Ng} \mathrm{C}$, et al. Optimal scanning protocols of 64-slice CT angiography in coronary artery stents: $\mathrm{An}$ in vitro phantom study. Eur Radiol. 2010;74(1):156-160.

19. Hickethier T, Baeßler B, Kroeger JR, et al. Monoenergetic reconstructions for imaging of coronary artery stents using spectral detector CT: In-vitro experience and comparison to conventional images. J Cardiovasc Comput. 2017;11(1):33-39.

20. Stettler C, Wandel S, Allemann S, et al. Outcomes associated with drug-eluting and baremetal stents: a collaborative network meta-analysis. The Lancet. 2007;370(9591):937-948.

21. Smits PC, Vlachojannis GJ, McFadden EP, et al. Final 5-year follow-up of a randomized controlled trial of everolimus-and paclitaxel-eluting stents for coronary revascularization in daily practice: the COMPARE trial (A Trial of Everolimus-Eluting Stents and Paclitaxel Stents for Coronary Revascularization in Daily Practice). JACC: Cardiovascular Interventions. 2015;8(9):1157-1165.

22. Bossi I, Klersy C, Black AJ, et al. In-stent restenosis: long-term outcome and predictors of subsequent target lesion revascularization after repeat balloon angioplasty. J Am Coll Cardiol. 2000;35(6):1569-1576.

23. Chen MS, John JM, Chew DP, et al. Bare metal stent restenosis is not a benign clinical entity. Am Heart J. 2006;151(6):1260-1264.

24. Alraies MC, Darmoch F, Tummala R, et al. Diagnosis and management challenges of instent restenosis in coronary arteries. World J Cardiol. 2017;9(8):640-651.

25. Min JK, Hasegawa JT, Machacz SF, et al. Costs and clinical outcomes for non-invasive versus invasive diagnostic approaches to patients with suspected in-stent restenosis. Int $J$ Cardiovasc Imaging. 2016;32(2):309-315. 
Tables

Table 1: Parameters of the used stents.

\begin{tabular}{|c|c|c|c|}
\hline Stent & Material & Strut thickness [mm] & Length [mm] \\
\hline $\begin{array}{l}\text { Chrono } \\
\text { (Sorin Biomedical) }\end{array}$ & $\begin{array}{l}\text { Cobalt-chrome } \\
\text { (CoCr) }\end{array}$ & 0.08 & 20 \\
\hline $\begin{array}{l}\text { Endeavor } \\
\text { (Medtronic) }\end{array}$ & CoCr & 0.091 & 30 \\
\hline $\begin{array}{l}\text { Prokinetic } \\
\text { (Biotronik) }\end{array}$ & $\mathrm{CoCr}$ & 0.06 & 15 \\
\hline $\begin{array}{l}\text { Radius } \\
\text { (Boston Scientific) }\end{array}$ & Nitinol & 0.085 & 20 \\
\hline $\begin{array}{l}\text { Omega } \\
\text { (Boston Scientific) }\end{array}$ & $\begin{array}{l}\text { Platinum Chromium } \\
\text { (PICr) }\end{array}$ & 0.081 & 16 \\
\hline $\begin{array}{l}\text { Promus Element Plus } \\
\text { (Boston Scientific) }\end{array}$ & $\mathrm{PICr}$ & 0.081 & 19 \\
\hline $\begin{array}{l}\text { Coroflex Please } \\
\text { (Braun) }\end{array}$ & Stainless Steel 316L & 0.12 & 19 \\
\hline $\begin{array}{l}\text { Tenax XR } \\
\text { (Biotronik) }\end{array}$ & Stainless Steel 316L & 0.08 & 15 \\
\hline $\begin{array}{l}\text { Tantal Coronary } \\
\text { (Abbott/Guidant) }\end{array}$ & Tantalum & 0.58 & 19 \\
\hline $\begin{array}{l}\text { Wiktor } \\
\text { (Medtronic) }\end{array}$ & Tantalum & 0.064 & 30 \\
\hline
\end{tabular}


Table 2: CT acquisition and reconstruction parameters of both systems. Since SPCCT was a research prototype not every option of commercial scanners was available and the DLCT parameters had to be adapted to it.

\begin{tabular}{|c|c|c|}
\hline & SPCCT & DLCT \\
\hline Scan Type & Axial & Axial \\
\hline Collimation & $9 \times 0.25 \mathrm{~mm}$ & $64 \times 0.625 \mathrm{~mm}$ \\
\hline Focal Spot Resolution & High & Standard \\
\hline Slice Thickness & $0.25 \mathrm{~mm}$ & $0.67 \mathrm{~mm}$ \\
\hline Rotation Time & $1 \mathrm{~s}$ & $0.27 \mathrm{~s}$ \\
\hline Voltage & $120 \mathrm{kV}$ & $120 \mathrm{kV}$ \\
\hline Current & $100 \mathrm{~mA} / / 100 \mathrm{mAs}$ & $368 \mathrm{~mA} / / 100 \mathrm{mAs}$ \\
\hline Dose Modulation & None & None \\
\hline Image Matrix & $512 \times 512$ & $512 \times 512$ \\
\hline Field of View & $102 \mathrm{~mm}$ & $102 \mathrm{~mm}$ \\
\hline Reconstruction Filter & Sharp & CD (Cardiac Detailed Stent) \\
\hline Iterative & No & Spectral Level 0) \\
\hline Reconstruction & & \\
\hline
\end{tabular}

CT: computed tomography; SPCCT: spectral photon counting CT; DLCT: dual-layer CT 
Table 3: Visible diameter of the in-stent and residual lumen averaged for both raters.

\begin{tabular}{|l|l|l|l|l|}
\hline Reconstruction & \multicolumn{2}{|c|}{ Visible lumen diameter [mm] } & \multicolumn{2}{c|}{ Residual lumen [mm] } \\
\hline & SPCCT & DLCT & SPCCT & DLCT \\
\hline PolyE & $1.60 \pm 0.59$ & $1.35 \pm 0.40$ & $0.45 \pm 0.32$ & 0 \\
\hline MonoE 40 & $1.80 \pm 0.17$ & $1.50 \pm 0.31$ & $0.44 \pm 0.31$ & 0 \\
\hline MonoE 60 & $1.61 \pm 0.60$ & $1.39 \pm 0.35$ & $0.43 \pm 0.31$ & 0 \\
\hline MonoE 90 & $1.53 \pm 0.59$ & $1.10 \pm 0.52$ & $0,36 \pm 0.27$ & 0 \\
\hline MonoE 120 & $1.46 \pm 0.58$ & $0.94 \pm 0.54$ & $0.14 \pm 0.21$ & 0 \\
\hline
\end{tabular}

SPCCT: spectral photon counting computed tomography; DLCT: dual-layer computed tomography

\begin{tabular}{|l|l|l|l|l|}
\hline Reconstruction & \multicolumn{2}{|c|}{ Image noise [HU] } & \multicolumn{2}{l|}{ Stenosis density difference [HU] } \\
\hline & SPCCT & DLCT & SPCCT & DLCT \\
\hline PolyE & $41.36 \pm 7.18$ & $37.29 \pm 7.04$ & $345.13 \pm 17.75$ & $356.68 \pm 190.08$ \\
\hline MonoE 40 & $158.47 \pm 24.29$ & $35.79 \pm 9.31$ & $667.16 \pm 49.52$ & $1037.88 \pm 257.26$ \\
\hline MonoE 60 & $44.26 \pm 8.70$ & $28.96 \pm 5.15$ & $355.17 \pm 26.41$ & $489.34 \pm 83.28$ \\
\hline MonoE 90 & $79.88 \pm 12.78$ & $28.27 \pm 4.68$ & $221.66 \pm 39.74$ & $289.01 \pm 159.86$ \\
\hline MonoE 120 & $98.79 \pm 15.48$ & $28.45 \pm 4.73$ & $173.42 \pm 41.72$ & $226.37 \pm 167.77$ \\
\hline
\end{tabular}

Table 4: Quantitative image quality parameters.

SPCCT: spectral photon counting computed tomography; DLCT: dual-layer computed tomography

Table 5: CNR values and subjective scores of both systems.

\begin{tabular}{|l|l|l|l|l|}
\hline Reconstruction & \multicolumn{2}{|c|}{ CNR } & \multicolumn{2}{c|}{ Subjective Score } \\
\hline & SPCCT & DLCT & SPCCT & DLCT \\
\hline PolyE & $9.00 \pm 3.22$ & $10.79 \pm 4.86$ & $4.10 \pm 1.52$ & $2.80 \pm 0.42$ \\
\hline MonoE 40 & $4.46 \pm 1.30$ & $31.58 \pm 12.54$ & $4.25 \pm 1.03$ & $2.75 \pm 0.23$ \\
\hline MonoE 60 & $8.42 \pm 2.17$ & $17.08 \pm 4.03$ & $3.95 \pm 1.46$ & $2.50 \pm 0.71$ \\
\hline
\end{tabular}




\begin{tabular}{|l|l|l|l|l|} 
MonoE 90 & $2.95 \pm 1.05$ & $9.36 \pm 2.63$ & $2.95 \pm 1.12$ & $2.15 \pm 0.71$ \\
\hline MonoE 120 & $1.88 \pm 0.27$ & $6.62 \pm 2.42$ & $2.25 \pm 0.72$ & $1.90 \pm 0.62$ \\
\hline
\end{tabular}

CNR: contrast-to-noise ratio; SPCCT: spectral photon counting computed tomography; DLCT: dual-layer computed tomography

Suppl. Table 1: Attenuation outside ( $\mathrm{ROI} 1)$ and inside $(\mathrm{ROI} 2)$ the Stent for the different reconstructions of SPCCT and DLCT.

\begin{tabular}{|l|l|l|l|l|}
\hline Reconstruction & \multicolumn{2}{|c|}{ ROI 1 } & \multicolumn{2}{c|}{ ROI 2 } \\
\hline & SPCCT & DLCT & SPCCT & DLCT \\
\hline PolyE & $399.83 \pm 6.02$ & $547.32 \pm 15.51$ & $449.90 \pm 57.93$ & $616.52 \pm 41.93$ \\
\hline MonoE 40 & $775.44 \pm 24.54$ & $1428.76 \pm 52.38$ & $842.75 \pm 50.41$ & $1523.54 \pm 68.00$ \\
\hline MonoE 60 & $407.14 \pm 4.28$ & $624.32 \pm 13.75$ & $467.62 \pm 65.52$ & $768.71 \pm 219.85$ \\
\hline MonoE 90 & $226.73 \pm 15.58$ & $257.62 \pm 18.23$ & $280.91 \pm 68.04$ & $429.48 \pm 296.74$ \\
\hline MonoE 120 & $181.12 \pm 15.82$ & $146.92 \pm 22.65$ & $230.01 \pm 79.27$ & $325.42 \pm 320.86$ \\
\hline
\end{tabular}

SPCCT: spectral photon counting computed tomography; DLCT: dual-layer computed tomography 


\section{Figure Legends}

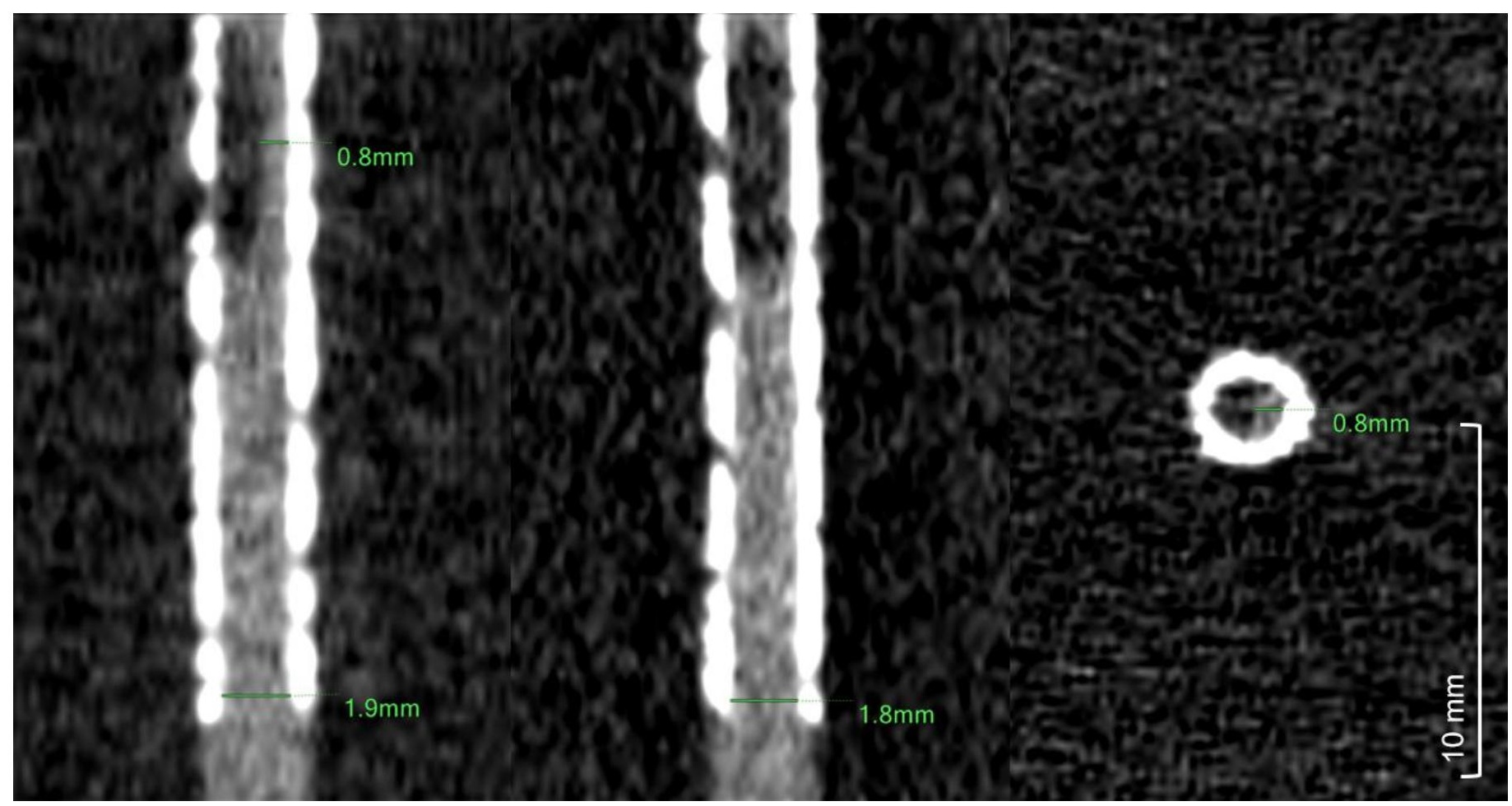

Figure 1: Coronal, sagittal and axial reconstruction of the Coroflex stent in spectral photon counting computed tomography PolyE images with measurements of the in-stent lumen and the residual lumen. The manual positioning of the artificial stenosis led to eccentric positions and therefore the residual lumen was averaged from the two largest measurements. 


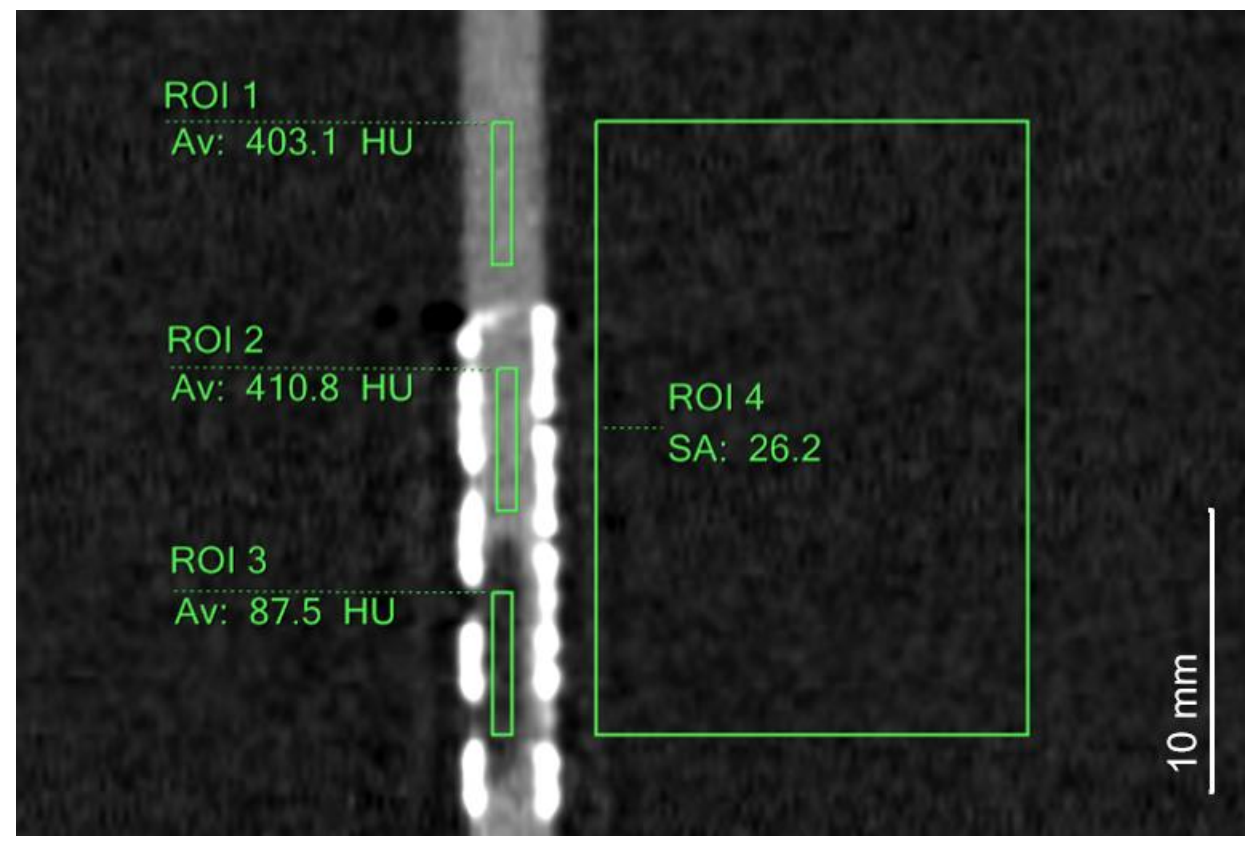

Figure 2: Measurement of the different regions of interest (ROI) in the PolyE image for the Chrono stent with spectral photon counting computed tomography. The first ROI was placed in the lumen of the artificial vessel. ROI 2 represents the attenuation of the contrast agent within the stent and ROI 3 within the stenosis. ROI 4 is outside of the artificial vessel to measure the overall image noise. 


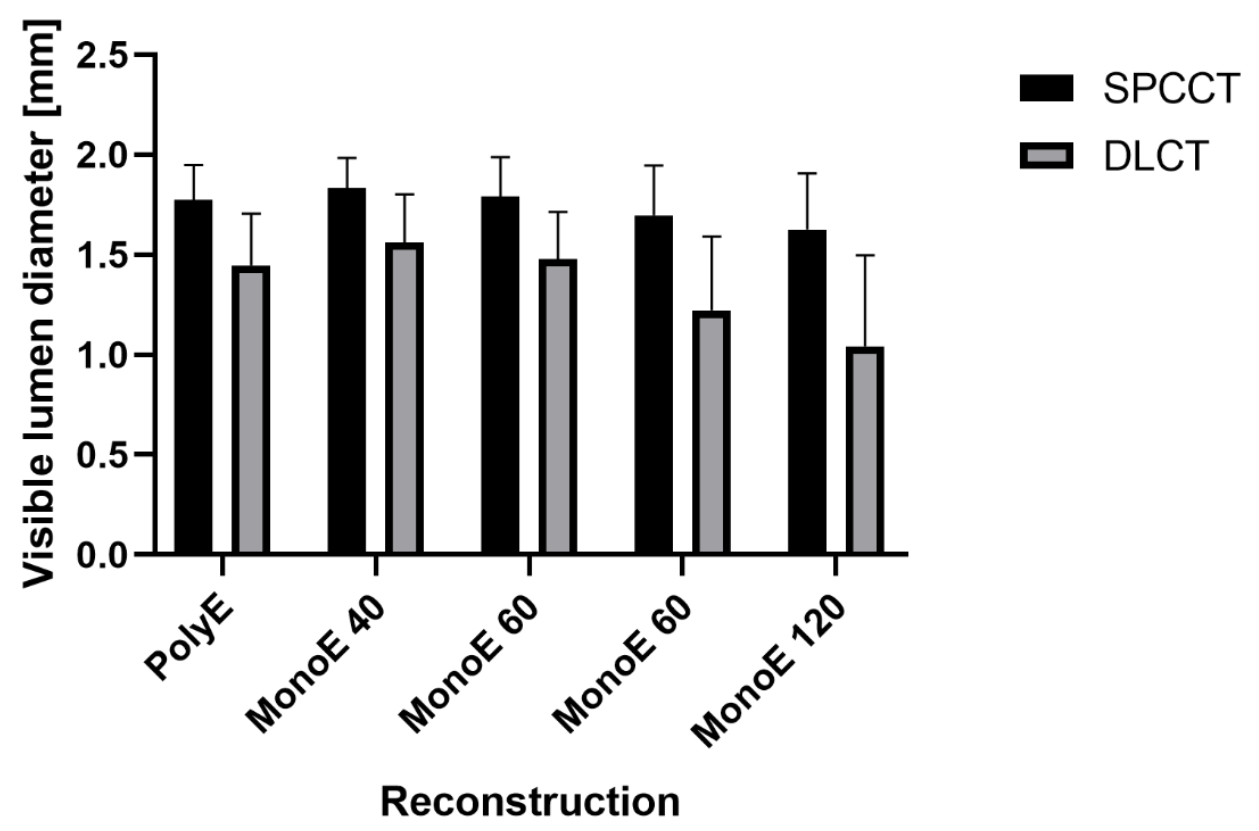

Figure 3: Diameter of the in-stent lumen. A clear trend towards better quantitative delineation of the stent diameter for spectral photon counting computed tomography compared to the dual-layer computed tomography, and for PolyE and low MonoE keV compared to high MonoE keV was observed without reaching statistical significance. 


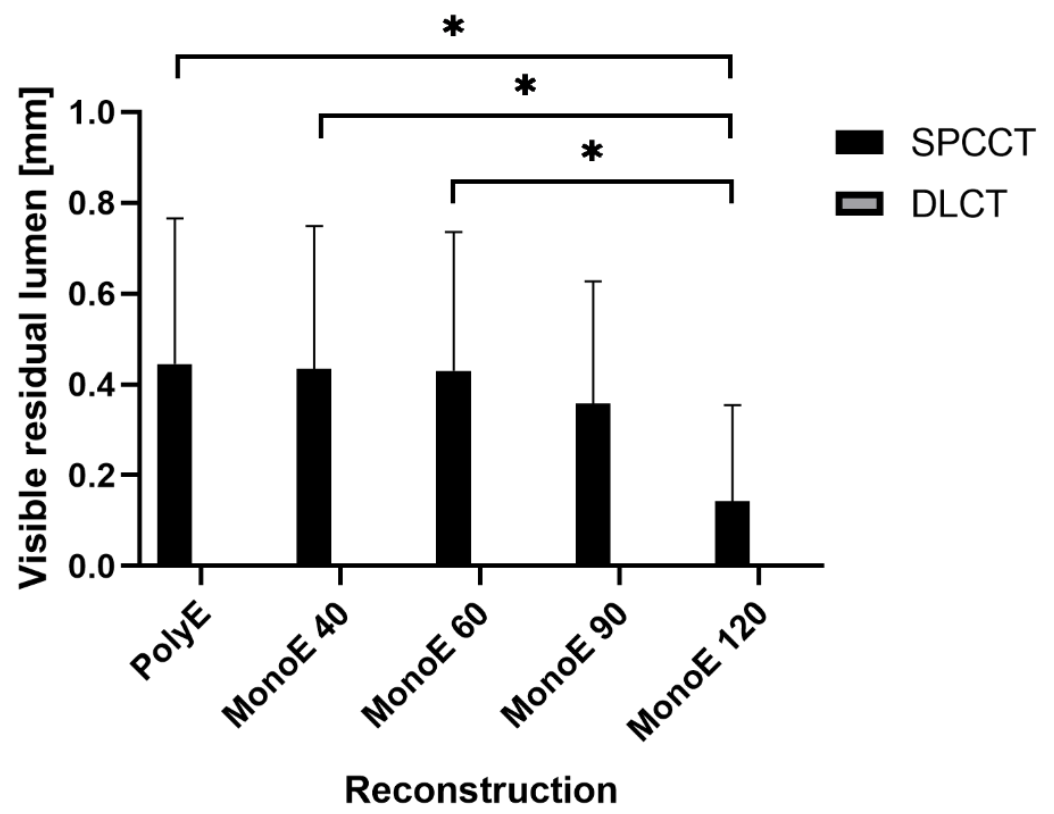

Figure 4: Residual lumen of all 10 stents. No residual lumen could be depicted for dual-layer computed tomography. The values for PolyE and lower keV images are comparable and significantly higher than for $120 \mathrm{keV}$ reconstructions (all $p<0.05)$. 


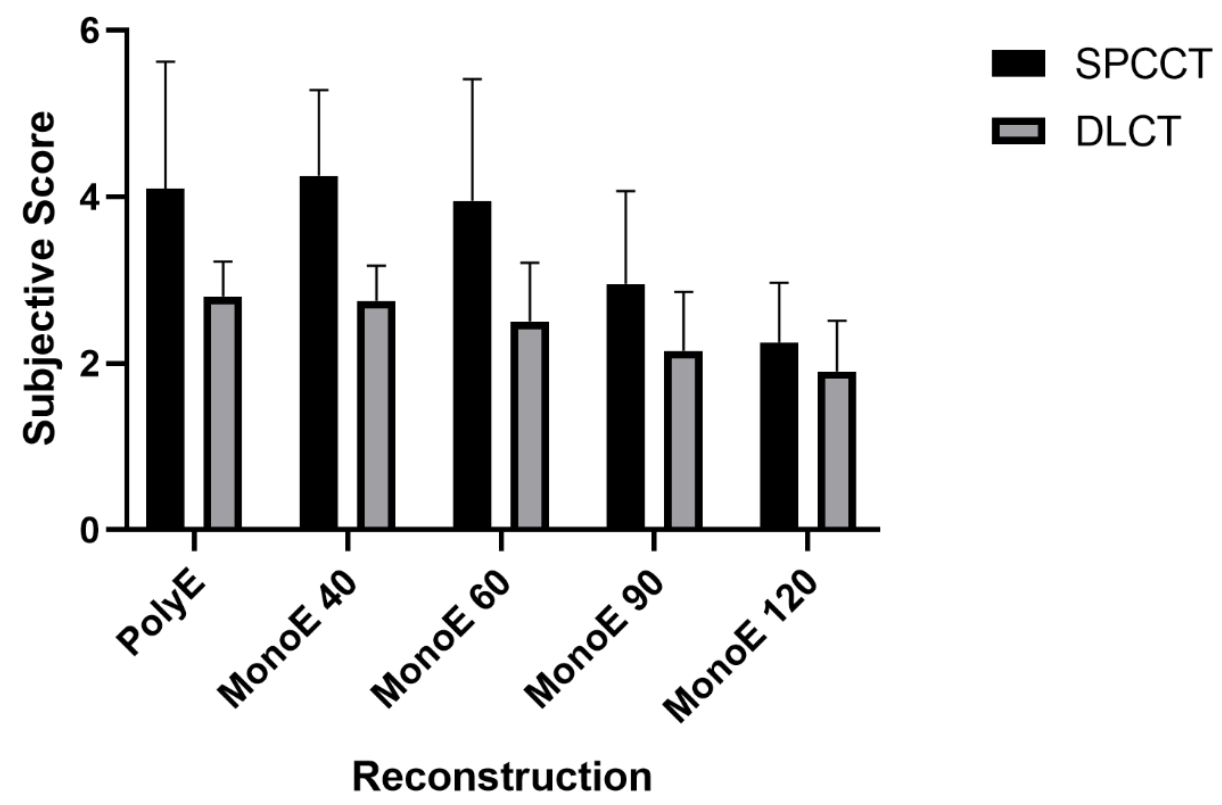

Figure 5: Subjective Scoring averaged for both raters. The spectral photon counting computed tomography (SPCCT) offered a significantly better image quality $(p<$ 0.0001) if averaged for all reconstructions with the best scores for MonoE 40 images. The ratings were higher for every SPCCT image compared to the corresponding dual-layer computed-tomography (DLCT) images. The PolyE, MonoE $40 \mathrm{keV}$ and MonoE $60 \mathrm{keV}$ reconstructions of the SPCCT images show significantly better subjective ratings than the MonoE $120 \mathrm{keV}$ SPCCT as well as the MonoE $90 \mathrm{keV}$ and $120 \mathrm{keV}$ DLCT images (all $\mathrm{p}<0.05$ ). 


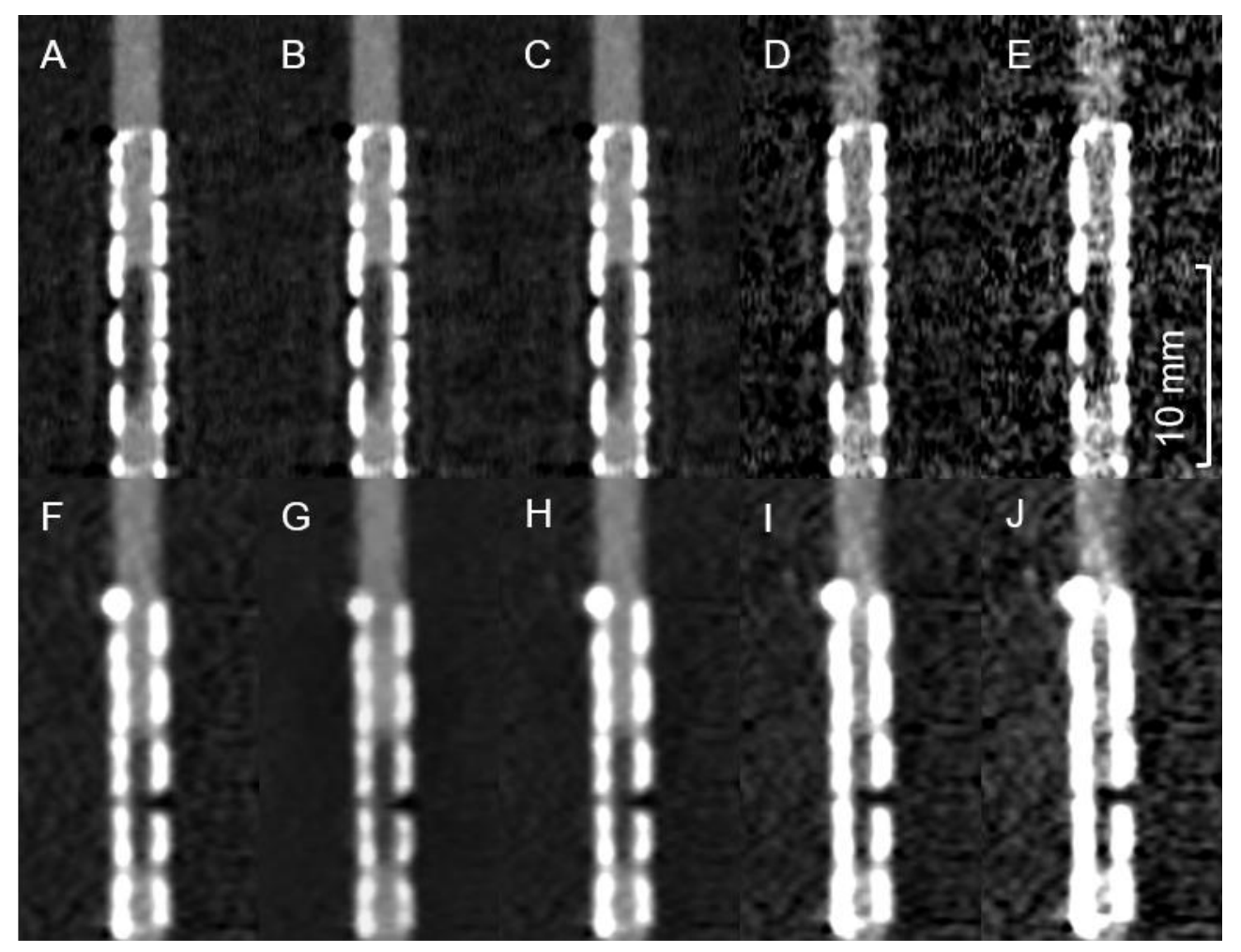

Figure 6: Comparison of the different reconstructions for the spectral photon counting computed tomography (A-E) and dual-layer computed tomography (F-J) with the Chrono stent and the individually adapted window settings. For both scanners, delineation of the stenosis was better for PolyE (A, F) and MonoE $40 \mathrm{keV}(\mathrm{B}, \mathrm{G})$ images compared to higher keV images (MonoE 60: C, H); MonoE 90: D, I) and especially MonoE $120 \mathrm{keV}(\mathrm{E}, \mathrm{J})$. The different window settings were optimized to delineate the stenosis but also influenced the artifact blooming and overall image noise. 